\title{
Slow Conformational Changes of the Voltage Sensor during the Mode Shift in Hyperpolarization-Activated Cyclic-Nucleotide-Gated Channels
}

\author{
Andrew Bruening-Wright and H. Peter Larsson \\ Neurological Sciences Institute, Oregon Health and Science University, Beaverton, Oregon 97006
}

\begin{abstract}
Hyperpolarization-activated cyclic-nucleotide-gated ( $\mathrm{HCN})$ channels are activated by hyperpolarizations that cause inward movements of the positive charges in the fourth transmembrane domain (S4), which triggers channel opening. If HCN channels are held open for prolonged times ( $>50 \mathrm{~ms}), \mathrm{HCN}$ channels undergo a mode shift, which in sea urchin ( $\mathrm{spHCN}$ ) channels induces a $>50 \mathrm{mV}$ shift in the midpoint of activation. The mechanism underlying the mode shift is unknown. The mode shift could be attributable to conformational changes in the pore domain that stabilize the open state of the channel, which would indirectly shift the voltage dependence of the channel, or attributable to conformational changes in the voltage-sensing domain that stabilize the inward position of $S 4$, thereby directly shifting the voltage dependence of the channel. We used voltage-clamp fluorometry to detect S4 movements and to correlate S4 movements to the different activation steps in spHCN channels. We here show that fluorophores attached to $\mathrm{S} 4$ report on fluorescence changes during the mode shift, demonstrating that the mode shift is not simply attributable to a stabilization of the pore domain but that $S 4$ undergoes conformational changes during the mode shift. We propose a model in which the mode shift is attributable to a slow, lateral movement in S4 that is triggered by the initial S4 gating-charge movement and channel opening. The mode shift gives rise to a short-term, activitydependent memory in $\mathrm{HCN}$ channels, which has been shown previously to be important for the stable rhythmic firing of pacemaking neurons and could significantly affect synaptic integration.
\end{abstract}

Key words: spHCN; S4; VCF; Ih; voltage hysteresis; mode shift

\section{Introduction}

Hyperpolarization-activated cyclic-nucleotide-gated (HCN) channels have been detected in a broad range of cells and have been suggested to play an important role in pacemaking, dendritic integration, synaptic transmission, controlling the restingmembrane properties, and reducing extreme hyperpolarizations (DiFrancesco, 1993; Pape, 1996). Knock-out experiments of HCN channels have resulted in mice with motor deficiencies, epilepsy, altered spatial learning, and heart arrhythmia, suggesting that HCN channels have widespread importance in the nervous system (Ludwig et al., 2003; Nolan et al., 2003; Schulze-Bahr et al., 2003; Ueda et al., 2004).

HCN channels belong to the superfamily of voltage-gated ion channels (Gauss et al., 1998). Most of the channels in this family, such as voltage-gated $\mathrm{K}^{+}$and $\mathrm{Na}^{+}$channels, are activated by an outward movement of positive charges in the fourth transmembrane domain (S4) in response to membrane depolarization (Hille et al., 1999). However, HCN channels open by inward

Received Aug. 31, 2006; revised Nov. 27, 2006; accepted Nov. 28, 2006.

This work was supported by American Heart Association Grant $0425914 Z$ (A.B.-W.) and National Institutes of Health Grant NS043259 (H.P.L.). We thank James Maylie, Fredrik Elinder, Jeff Karpen, and John Adelman for helpful comments on this manuscript and Sandra 0ster for editing this manuscript.

Correspondence should be addressed to H. Peter Larsson, Neurological Sciences Institute, Oregon Health \& Science University, 505 N.W. 185th Avenue, Beaverton, OR 97006. E-mail: larssonp@ohsu.edu.

D01:10.1523/JNEUROSCI.3801-06.2007

Copyright $\odot 2007$ Society for Neuroscience $\quad$ 0270-6474/07/270270-09\$15.00/0 movement of positive charges in $\mathrm{S} 4$ in response to membrane hyperpolarization (Männikkö et al., 2002; Bell et al., 2004; Vemana et al., 2004). The activation of HCN channels is also modulated by cyclic nucleotides that bind directly to the channel $\mathrm{C}$ terminus (Robinson and Siegelbaum, 2003). For the sea urchin HCN (spHCN) channel, lowering intracellular cAMP induces channel inactivation (Gauss et al., 1998).

Recently, it was shown that spHCN and mammalian HCN channels shift between two modes of voltage activation and that the two modes have very different midpoints of activation: $>50$ $\mathrm{mV}$ shift between modes for spHCN channels (Männikkö et al., 2005; Elinder et al., 2006) (see Fig. $1 A-C$ ). The relative occupancy of the two modes depends on the previous activity of the HCN channels, such that the channels are mainly in mode I at depolarized potentials and mode II at hyperpolarized potentials (see Fig. $1 A, B)$. The shifts between the two modes of voltage activation result in a voltage hysteresis in the ionic currents from $\mathrm{HCN}$ channels during rhythmic firing of pacemaker cells, i.e., the ionic currents turn on and off at different voltages during the up and down stroke of the action potential. This voltage hysteresis has been shown to be important for preventing arrhythmia in model pacemaker cells (Männikkö et al., 2005; Elinder et al., 2006).

The molecular mechanism underlying the mode shift is unknown. The mode shift occurs in excised cell-free patches, is relatively fast ( $\tau$ of $50-100 \mathrm{~ms}$ ), and is easily reversible, suggesting that it is attributable to intrinsic conformational changes in the 
HCN channel (Männikkö et al., 2005). Permeating and poreblocking ions modulate the time course of the mode shifts, suggesting that conformational changes in the pore play some role in the mode shift (Männikkö et al., 2005). However, it is unknown whether S4 plays an active role in the mode shifts.

In the studies reported here, we used voltage-clamp fluorometry (VCF) to detect conformational changes in S4 during the mode shift in the voltage dependence of spHCN channels (Mannuzzu et al., 1996). We found that fluorophores attached to outer S4 residues report on conformational changes and that these conformational changes correlate with the mode shifts in spHCN channels.

\section{Materials and Methods}

Expression system. Experiments were performed on sea urchin spHCN channels (provided by U. B. Kaupp, Forschungszentrum Julich, Julich, Germany) and mouse HCN1 channels heterologously expressed in $\mathrm{Xe}$ nopus oocytes. The HCN1 channels had a stop codon introduced at position S391 to remove the cyclic nucleotide-binding site (Wainger et al., 2001). Site-directed mutagenesis was performed on spHCN cDNA (in the pGEM-HE expression vector) using the QuikChange system (Stratagene, La Jolla, CA). The DNA was linearized with NheI before RNA synthesis and purification using a T7 mMessage mMachine kit (Ambion, Austin, TX). Fifty nanoliters of cRNA (ranging from 2.5 to 50 ng) were injected into each oocyte, and recordings were made by twoelectrode voltage clamp (TEVC) $2-5 \mathrm{~d}$ after injection.

Two-electrode voltage clamp. Whole-cell ionic currents were measured with the TEVC technique using a Geneclamp 500B (Molecular Devices, Palo Alto, CA). Microelectrodes were pulled using borosilicate glass, filled with a $3 \mathrm{M} \mathrm{KCl}$ solution; each microelectrode had a resistance between 0.5 and $1.5 \mathrm{M} \Omega$. All experiments were performed at room temperature. The bath solution contained the following (in $\mathrm{mm}$ ): $89 \mathrm{KCl}, 10$ HEPES, $0.4 \mathrm{CaCl}_{2}$, and $0.8 \mathrm{MgCl}_{2}$. The $\mathrm{pH}$ was adjusted to 7.4 with $\mathrm{KOH}$, yielding a final $\mathrm{K}$ concentration of $100 \mathrm{~mm}$. Data were digitized at $5 \mathrm{kHz}$ (Digidata 1322A), filtered at $1 \mathrm{kHz}$, and monitored and collected using pClamp software (Molecular Devices).

Voltage-clamp fluorometry. For VCF experiments, single cysteines were introduced in the $\mathrm{S} 4$ of spHCN channels by site-directed mutagenesis. Oocytes expressing either the cysteine-containing or wild-type (wt) spHCN channels were incubated for 5-30 min (typically $20 \mathrm{~min}$ to saturate binding to the introduced cysteine) in a bath solution that was supplemented with $100 \mu \mathrm{M}$ of the cysteine-labeling Alexa-488 C5-maleimide (Invitrogen, Carlsbad, CA). Oocytes expressing the single-cysteine spHCN channels usually labeled two to five times more than uninjected control oocytes, whereas oocytes expressing wt spHCN channels did not label significantly more than uninjected control oocytes, showing that there was insignificant labeling of endogenous cysteines in spHCN channels. After washing to remove any unbound Alexa-488 C5-maleimide, fluorescently labeled oocytes were placed animal-pole "up" in a bath housed on a Leica (Nussloch, Germany) DMLFS upright fluorescence microscope. Light was focused on the animal pole of the oocyte through a $20 \times$ objective and was passed through a filter cube from Chroma Technology (Brattleboro, VT) 41026 (HQ495/30×, Q515LP, HQ545/ $50 \mathrm{~m})$. The oocytes were voltage clamped with the TEVC technique, and the current and the fluorescence were recorded simultaneously. Fluorescence signals were low-pass Bessel filtered (Frequency Devices, Haverhill, $\mathrm{MA})$ at $200 \mathrm{~Hz}$ and digitized at $1 \mathrm{kHz}$. In VCF, the changes in fluorescence are assumed to be attributable to local protein conformational changes that alter the environment of the fluorophore, thereby altering the fluorescence intensity or spectrum of the fluorophore. VCF has been shown previously to detect conformational changes in a number of ion channels and transporters (Mannuzzu et al., 1996; Cha and Bezanilla, 1997; Li and Lester, 2002; Larsson et al., 2004).

Modeling. With modeling, we here want to show that both the voltage shift in the gating charge versus voltage curve, $Q(V)$ (Fig. 1A), and the conductance versus voltage curve, $G(V)$ (Fig. $1 B$ ), associated with the mode shift in spHCN channels (Männikkö et al., 2005) can be caused by (1) a conformational change in the pore that changes the energetics of pore opening, (2) a conformational change in the voltage-sensing domain that changes the energetics of S4 movement, or (3) both. Model 1 shows a simple, two-mode model for HCN channels with separate S4 (L) and gate $(\mathrm{K})$ transitions. It reproduces the voltage shifts of the $Q(V)$ and the $G(V)$ that are associated with the mode shift $(\mathrm{M})$ transition in HCN channels (Männikkö et al., 2005):

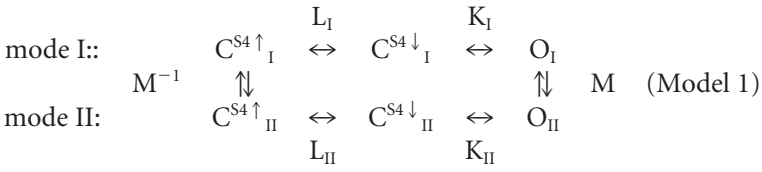

$$
\begin{aligned}
& \mathrm{L}_{i}=e^{-\left(q V-W_{\mathrm{cc}}[i]\right) / k T}, \\
& \mathrm{~K}_{i}=e^{-W_{\mathrm{co}}[i] / \mathrm{kT}},
\end{aligned}
$$

$i=$ mode I or mode II, where $W_{\mathrm{cc}}[i]$ and $W_{\mathrm{co}}[i]$ are the free energies between the states $\mathrm{C}^{\mathrm{S} 4 \uparrow}$ and $\mathrm{C}^{\mathrm{S} \downarrow} \downarrow$ (at $V$ of $0 \mathrm{mV}$ ) and states $\mathrm{C}^{\mathrm{S} 4 \downarrow}$ and $\mathrm{O}$ (open channel), respectively. The $\mathrm{L}$ transitions are the only voltagedependent transitions in the model, and $q$ is the equivalent charge moved by the voltage sensor S4 between state $\mathrm{C}^{\mathrm{S} 4} \uparrow$ (closed channel with S4 in the resting state) and $\mathrm{C}^{S 4 \downarrow}$ (closed channel with $\mathrm{S} 4$ in the activated state). The equilibrium constant $\mathrm{M}$ for the mode shift was previously assigned a value of 10.9 (Männikkö et al., 2005).

The mode shift transitions have been shown to be slower than the S4 movement or the opening of the channel (Männikkö et al., 2005). Therefore, the open probability in a mode can be approximated by an expression that only considers the states in that mode (because the transitions between states in the same mode are faster than transitions between states in different modes):

$$
p^{\text {open }}[i]=1 /\left(1+1 / \mathrm{K}_{i}+1 /\left(\mathrm{K}_{i} \mathrm{~L}_{i}\right)\right) .
$$

HCN channels have been shown to have a high maximal open probability, $p^{\text {open }},(>0.85)$ (Johnson and Zagotta, 2005), which means that $1 / \mathrm{K} \ll 1$. Therefore, we simplified Equation 3 and derived an expression that relates the voltage shift between the two modes, $\Delta V_{1 / 2}$, to the changes in $W_{\mathrm{cc}}$ and $W_{\mathrm{co}}$ between the two modes:

$p^{\text {open }}[i]=1 /\left(1+1 /\left(\mathrm{K}_{i} \mathrm{~L}_{i}\right)\right)=1 /\left(1+e^{+\left(q V-W_{\mathrm{cc}}[i]-\mathrm{W}_{\mathrm{co}}[i]\right) / k T}\right)=$

$$
1 /\left(1+e^{+q\left(V-V_{1 / 2}[i]\right) / k T}\right),
$$

$$
\text { where } V_{1 / 2}[i]=\left(W_{c c}[i]+W_{c o}[i]\right) / q \text {, }
$$

$$
\Delta V_{1 / 2}=\left(\Delta W_{\mathrm{cc}}+\Delta W_{\mathrm{co}}\right) / q \text {. }
$$

It is clear from Equation 6 that a change in $V_{1 / 2}$ between mode I and mode II can be attributable to a change in the energetics of the closed to open transition, $\Delta W_{\text {co }}$ (Fig. $1 D$ ), in the energetics of the voltage sensor movement, $\Delta W_{c c}$ (Fig. $1 E$ ), or in both (Fig. $1 F$ ). With the same assumptions, an identical expression (Eq. 6) can be derived for the shift in the $Q(V)$. Therefore, both the shift in the $Q(V)$ and the $G(V)$ during the mode shift in spHCN channels (Männikkö et al., 2005) could be attributable to a change in only one of the two transitions, for example in the pore opening $\left(\Delta W_{\text {co }}\right)$.

Modeling for Figures 5 and 6 were done using a two-mode, 20-state HCN channel model (A. Bruening-Wright, F. Elinder, and H. P. Larsson, unpublished observations). This model is similar to the two-mode model 1 , but it has four independent S4s that can move in both open and closed channels. The current and voltage traces were simulated using Berkeley Madonna (University of California, Berkeley, Berkeley, CA). For the voltage simulations (see Fig. 6), a simple, passive cell model was used, because all voltages were assumed to be below the threshold for action potentials: $d V / d t=-1 / C_{\mathrm{m}} \times\left(G_{\text {leak }} \times\left(V-V_{\text {leak }}\right)+I_{\text {synaptic }}+I_{\mathrm{HCN}}\right)$, where $C_{\mathrm{m}}$ is $25 \mathrm{pF}, G_{\text {leak }}$ is $1 \mathrm{nS}$, and $V_{\text {leak }}$ is $-80 \mathrm{mV}$.

\section{Results}

There are three major classes of mechanisms, intrinsic to the channel protein, that could generate a shift in the voltage dependence during the mode shift (Fig. 1): (1) conformational changes 

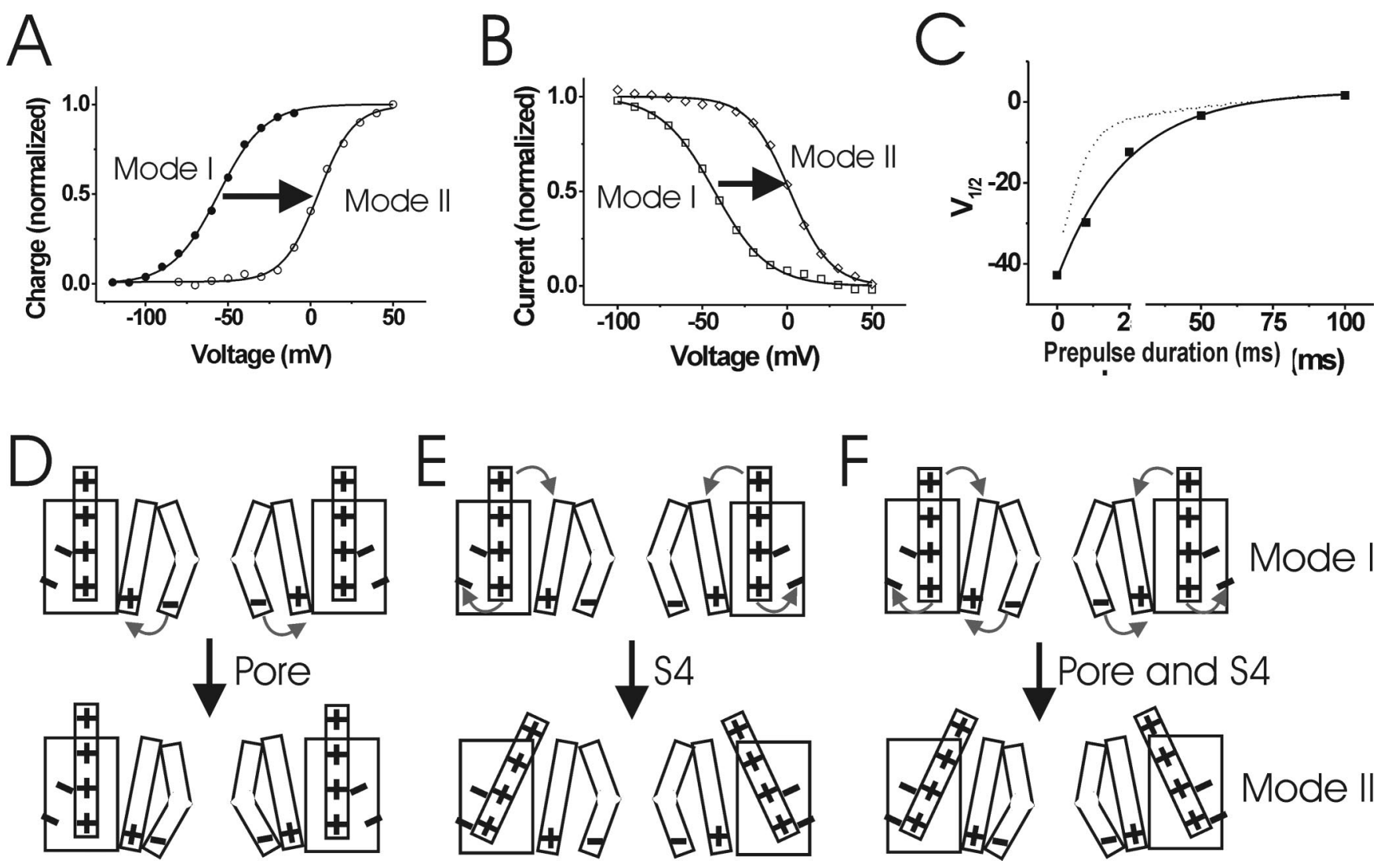

Figure 1. Different models for generating shifts in the voltage dependence of HCN channels. Experimental shifts in the midpoints of the $Q(V)(A)$ and $G(V)(B)$ accompanying the mode shift in spHCN channels (Männikkö et al., 2005), induced by voltage steps to $-80 \mathrm{mV}$ for $100 \mathrm{~ms} . A, Q(V)$ measured from a holding potential of $-10 \mathrm{mV}(\mathbf{O})$ and $-80 \mathrm{mV}(O)$ for nonconducting P435Y spHCN channels. $B, G(V)$ measured as isochronal tail currents at $+50 \mathrm{mV}$ after equilibration at the indicated voltage for $80 \mathrm{~ms}$ with ( $\diamond)$ or without $(\square)$ a $100 \mathrm{~ms}$ prepulse to $-80 \mathrm{mV}$. Holding potential was $0 \mathrm{mV}$. The $Q(V)$ and $G(V)$ were well fit by Boltzmann curves, i.e., $G(V)=1 /\left(1+\exp \left(q\left(V-V_{1 / 2}\right) / k T\right)\right)$. $C$, Increasing lengths of the prepulse to $-80 \mathrm{mV}$ increased the size of the $G(V)$ shift $(\square)$. The $G(V)$ shift is measured as the shift in the midpoint of activation, $V_{1 / 2}$ as determined in $\boldsymbol{B}$. The time course for the $G(V)$ shift was slower than the onset of the ionic current during the prepulse (dotted line), showing that the mode shift is slower than channel opening. $\boldsymbol{D}-\boldsymbol{F}, A$ change in $V_{1 / 2}$ may be attributable to a change in the free energy of opening of the activation gate $\Delta W_{\text {co }}$ $(\boldsymbol{D})$, a change in the free energy of $\$ 4$ movement $\Delta W_{c c}(\boldsymbol{E})$, or a change in both $(\boldsymbol{F})$ (for energy calculations, see Materials and Methods, Modeling). $\boldsymbol{D}$, A putative conformational change in the pore domain (shown here as an increased electrostatic interaction between $S 5$ and $S 6$ ) that stabilizes the open conformation would shift the voltage dependence by changing $\Delta W_{c 0}$. $E$, A putative conformational change in the voltage sensor domain (shown here as an increased electrostatic interaction between S2 and S4) that stabilizes the open conformation would shift the voltage dependence by changing $\Delta W_{c c} F$, A putative global conformational change of whole channel protein that stabilizes the open conformation would shift the voltage dependence by changing both $\Delta W_{\text {co }}$ and $\Delta W_{\text {cc }}$.

in the pore domain that stabilize the open state of the channel, which indirectly shift the voltage dependence of the channel (Fig. $1 D),(2)$ conformational changes in the voltage-sensing domain that stabilize the inward position of S4, thereby directly shifting the voltage dependence of the channel (Fig. 1E), or (3) a global conformational change of the whole protein that directly stabilizes both $\mathrm{S} 4$ and the pore (Fig. $1 F$ ). In this study, we used VCF to test whether S4 undergoes conformational changes during the mode shift or whether the mode shift is only attributable to an indirect allosteric effect of the pore on the voltage sensing of $\mathrm{HCN}$ channels. In VCF, a fluorophore is attached to the channel, and the fluorescence and the current from the fluorescently labeled channel are recorded simultaneously under voltage clamp. Fluorescence changes from the fluorophore are assumed to be attributable to conformational changes, in the region to which the fluorophore is attached, that alter the environment of the fluorophore, thereby altering the fluorescence intensity or spectrum of the fluorophore. VCF has been shown previously to detect conformational changes in a number of ion channels and transporters (Mannuzzu et al., 1996; Cha and Bezanilla, 1997; Li and Lester, 2002; Larsson et al., 2004).
Fluorescence from residue 326 reports on conformational changes that are slower than channel opening

To measure conformational changes in S4 during HCN channel activity, we introduced a cysteine at position 326 in the $\mathrm{N}$-terminal region of S4 (Fig. $2 \mathrm{~A}$ ). We labeled the cysteine with the cysteine-specific fluorophore Alexa-488 C5-maleimide and used VCF to monitor current and fluorescence simultaneously under TEVC. Wild-type spHCN channels exposed to the same fluorescence-labeling conditions as $326 \mathrm{C}$ channels did not display any significant fluorescence signals, suggesting that endogenous cysteines did not contribute to the fluorescence signals (Fig. $2 B$ ). The currents from Alexa-488-labeled 326C spHCN channels were similar to the currents from wild-type spHCN channels, except that the $G(V)$ for the Alexa-488-labeled 326C channels was shifted by $-30 \mathrm{mV}$ compared with in wildtype channels as has been reported previously for 326C channels (Männikkö et al., 2002). Figure $2 C$ shows the current and fluorescence from Alexa-488-labeled 326C channels. For channels labeled at 326C, the midpoint of the $G(V)$ curve was similar to the midpoint for the fluorescence versus voltage $[F(V)]$ curve $(G$ : $V_{1 / 2}$ of $-73.8 \pm 6.8 \mathrm{mV} ; F: V_{1 / 2}$ of $-76.0 \pm 1.5 \mathrm{mV} ; n=5$ ) (Fig. 


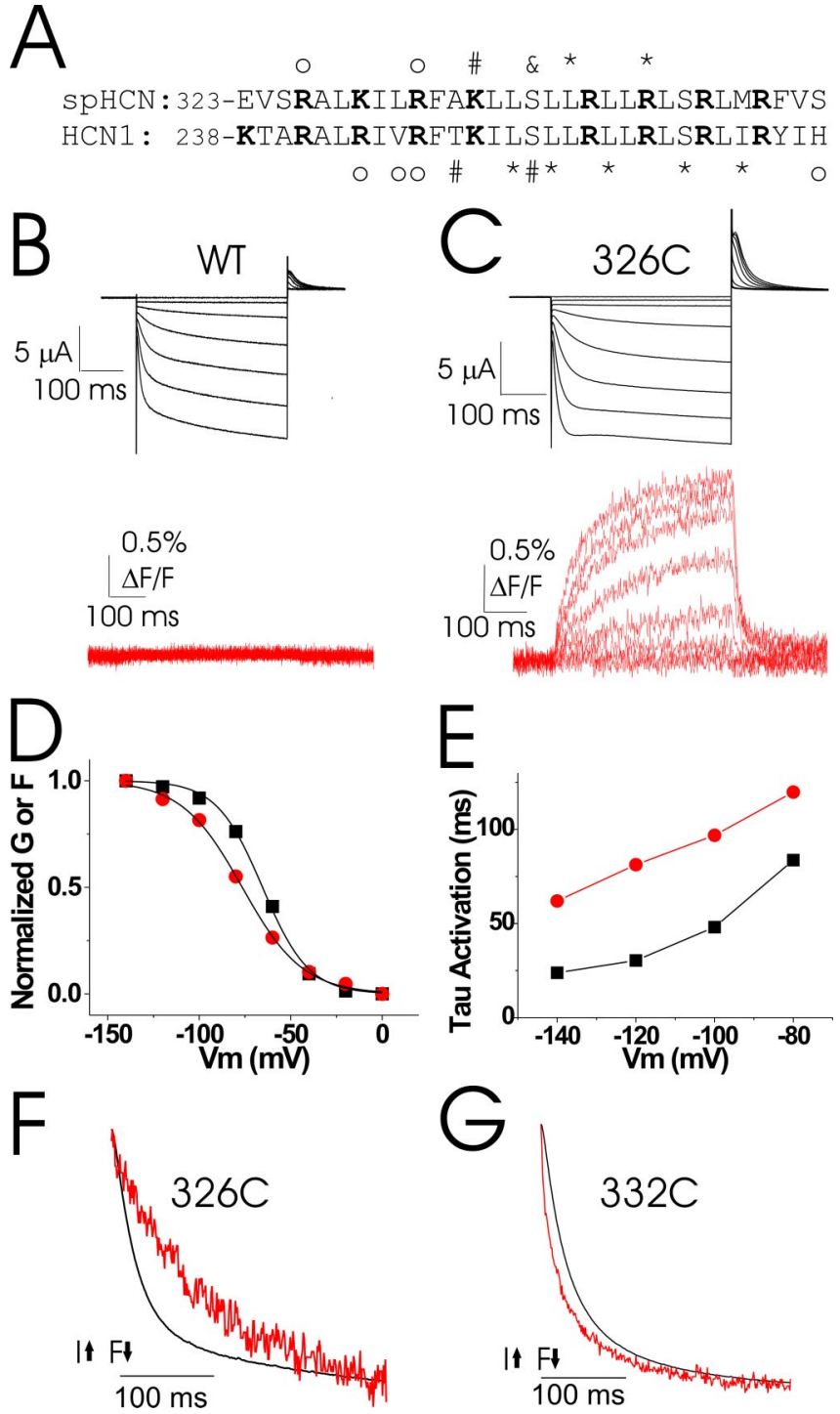

Figure 2. Fluorescence from $326 \mathrm{C}$ is slower than ionic current activation. $\boldsymbol{A}$, Sequence alignment of spHCN and HCN1 $\mathrm{S} 4$ domains. Marked residues exhibit state-dependent modification by intracellular $(*)$, extracellular (\#), or both intracellular and extracellular reagents $(\&)$, or state-independent modification by either intracellular or extracellular reagents (0) (Männikkö et al., 2002; Bell et al., 2004; Vemana et al., 2004). B, C, Representative current (top) and fluorescence (bottom) records from Alexa-488 (5-maleimide-exposed wt spHCN channels ( $\boldsymbol{B}$ ) and $326 \mathrm{C}$ channels $(C)$. Channels were held at $0 \mathrm{mV}$ and then stepped to negative potentials $(0$ to $-140 \mathrm{mV}$ ), followed by a step to $+50 \mathrm{mV}$. Note that $326 \mathrm{C}$ channel currents precede the fluorescence change and that wt channels do not show a fluorescence change. $\boldsymbol{D}, \boldsymbol{E}$, Steadystate $(\boldsymbol{D})$ and kinetic $(\boldsymbol{E})$ analysis of the voltage dependence of the conductance $(\boldsymbol{\square})$ and fluorescence (red circles) from $\boldsymbol{C}$. The fluorescence signal was slower than ionic current activation across a range of voltages. Average time constants (milliseconds) for fluorescence and ionic current were, respectively, as follows: $132.1 \pm 11.6$ and $75.6 \pm 15.1(-80 \mathrm{mV}) ; 98.5 \pm 5.1$ and $32.7 \pm 6.3(-100 \mathrm{mV}) ; 75.4 \pm 4.3$ and $22.3 \pm 3.8(-120 \mathrm{mV})$; and $61.1 \pm 4.2$ and $15.6 \pm 2.7(-140 \mathrm{mV}) ; p<0.01$ at each voltage. $\boldsymbol{F}, \boldsymbol{G}$, Scaled and overlaid current (black line) and fluorescence (red line) in response to a voltage step to $-120 \mathrm{mV}$ for $326 \mathrm{C}(\boldsymbol{F})$ and $332 \mathrm{C}(\boldsymbol{G})$ channels. Note that the fluorescence signal has been inverted for a direct kinetic comparison with the current traces. The positive direction of current $(\mathrm{I})$ and fluorescence $(\mathrm{F})$ is indicated by black arrows.

$2 D)$, whereas the activation of the ionic current was faster than the fluorescence changes over a range of activating voltages (Fig. $2 E$ ). For large hyperpolarizations, the ionic current clearly preceded the fluorescence signal, suggesting that the fluorescence from 326C-labeled channels reported on a conformational change that is distinct from and slower than gating-charge move- ment or channel opening (Fig. $2 F$ ). In contrast, fluorophores attached to $332 \mathrm{C}$, which is closer to the gating-charge carrying region of $\mathrm{S} 4$ (Fig. 2A), reported on a conformational change that was faster than channel opening, presumably the gating-charge movement of S4 (Fig. 2G).

We have shown previously that HCN channels undergo a mode shift that occurs on a timescale that is slower than the time course of the ionic current (Fig. 1C). One possibility is that 326Clinked fluorophores reported on conformational changes during the mode shift. Another possibility is that 326C-linked fluorophores reported on conformational changes during inactivation, which has also been shown to be slower than channel opening (Gauss et al., 1998).

Fluorescence from residue 326 correlates with the mode shift but not with inactivation

Under conditions with a low intracellular cAMP concentration, spHCN channels undergo a type of inactivation that is slower than channel activation (Gauss et al., 1998; Rothberg et al., 2002; Shin et al., 2004). To test whether the slow fluorescence signal from 326C channels during large hyperpolarizations correlates temporally with inactivation, we compared the time course of the fluorescence change with the time course of inactivation. The current from spHCN channels did not display any apparent inactivation in whole-cell recordings (Fig. $3 A$ ), presumably because the basal level of cyclic nucleotides in oocytes saturates the cyclic nucleotide-binding domain (CNBD) (Männikkö et al., 2002), but the spHCN channels could still undergo a small amount of inactivation that could generate the slow fluorescence changes (Shin et al., 2004). Therefore, it was necessary to design an spHCN channel that clearly inactivates in whole oocytes to compare the time course of inactivation with the time course of the slow fluorescence changes. We mutated R620, a residue in the CNBD of spHCN channels, to a glycine residue to weaken cyclic nucleotide binding (Chen et al., 2001; Ulens and Siegelbaum, 2003). This mutation clearly induced inactivation in R620G channels in whole oocytes (Fig. $3 B$ ). The inactivation was removed by an application of a high concentration $(1 \mathrm{mM})$ of a membrane-permeable cAMP analog, 8-(4-chlorophenylthio)adenosine $3^{\prime}, 5^{\prime}$-monophosphate (data not shown), suggesting that the inactivation induced by the R620G mutation is not attributable to a different mechanism than the cAMP-dependent inactivation in wt spHCN channels. The time constant of inactivation at $-120 \mathrm{mV}$ in $326 \mathrm{C} / 620 \mathrm{G}$ channels was $407.8 \pm 49.9 \mathrm{~ms}$ $(n=4)$, significantly slower than the fluorescence signal from 326C/620G channels $(121.3 \pm 13.1 \mathrm{~ms} ; n=4 ; p<0.01)$ (Fig. $3 D$ ), demonstrating that the fluorescence signals from $326 \mathrm{C}$ channels do not report on the inactivation of spHCN channels.

We demonstrated previously that changes in the ionic currents and the gating currents in response to different lengths of hyperpolarizing prepulses can be described by a two-mode model of activation of HCN channels, a model in which the channels first open in mode I and then undergo a slower mode-shift transition to mode II (Männikkö et al., 2005). We also showed that changes in the tail current kinetics (channels that are in mode I close with single-exponential time course, whereas channels in mode II close more slowly, with a sigmoidal time course) correlate well with the mode shifts (Männikkö et al., 2005).

To test whether the slow fluorescence signal from 326C channels during large hyperpolarizations correlates temporally with the mode shifts, we measured the time course of the change in the tail currents after activating prepulses of increasing duration (Fig. $3 E)$. We have shown previously that, after short prepulses, $\mathrm{HCN}$ 


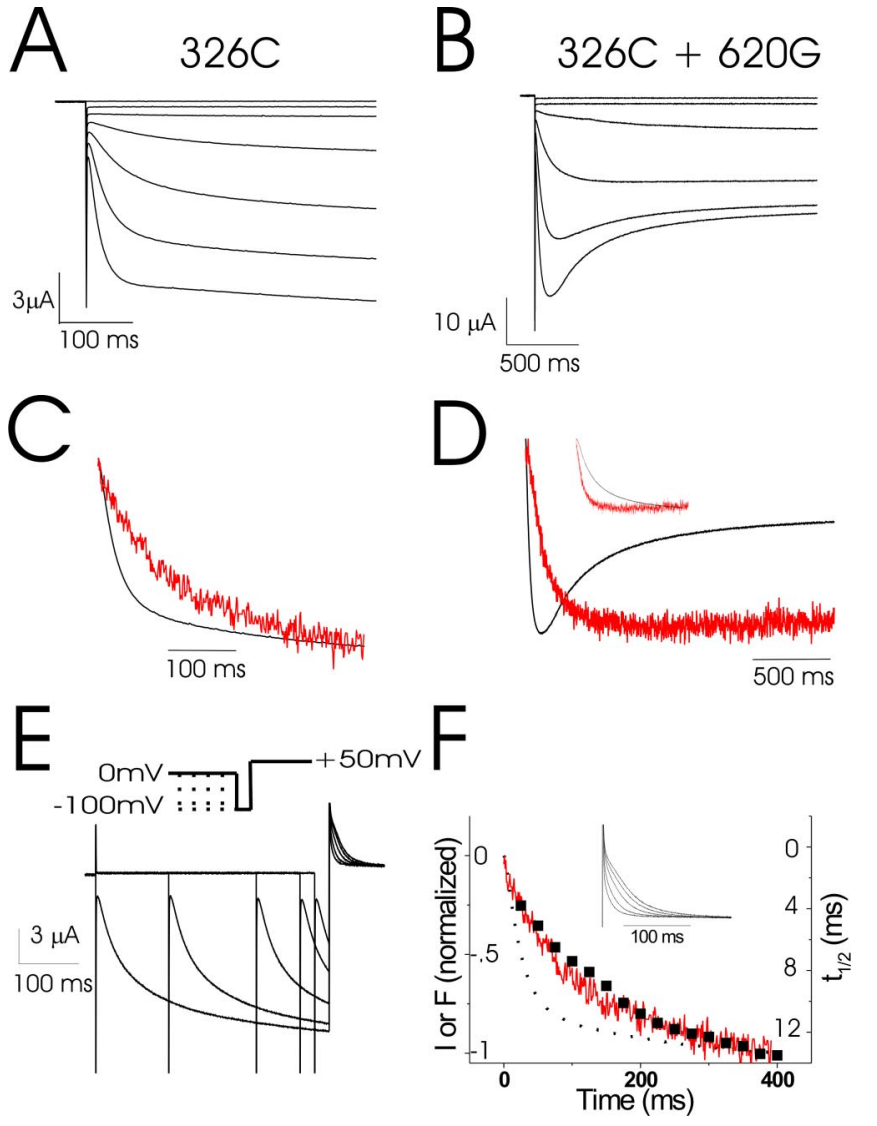

Figure 3. Fluorescence from $326 \mathrm{C}$ channels correlates with the mode shift but not inactivation. $A, B$, Currents in response to voltage steps from 0 to $-120 \mathrm{mV}$, in $20 \mathrm{mV}$ increments from Alexa-488-labeled 326C channels that do not show any apparent cAMP-dependent inactivation (A) and from Alexa-488-labeled 326C/620G channels that do exhibit cAMP-dependent inactivation $(\boldsymbol{B}) . \boldsymbol{C}, \boldsymbol{D}$, Scaled and overlaid current (black line) and fluorescence (red line) in response to a voltage step to $-120 \mathrm{mV}$ for non-inactivating $326 \mathrm{C}$ channels $(C)$ and inactivating $326 \mathrm{C} /$ $620 \mathrm{G}$ channels (D) (inset, the inactivation component of the current trace scaled and overlaid with the fluorescence change). The time constants for the currents were $28 \mathrm{~ms}$ for activation and $360 \mathrm{~ms}$ for inactivation, whereas the time constant for the fluorescence change was $105 \mathrm{~ms}$. $\boldsymbol{E}$, Development of a delay in the tail currents at $+50 \mathrm{mV}$ after increasing lengths of a $-100 \mathrm{mV}$ prepulse for Alexa-488-labeled 326C channels. Inset, Voltage protocol used. $\boldsymbol{F}$, Time course of the development of the tail-current delay (from $\boldsymbol{E}$ ), measured as the time $t_{1 / 2}(\square)$ for the tail-current amplitude to decay $50 \%$. The ionic current (dotted line) and the fluorescence (red line) during the prepulse are shown for comparison. Note that the fluorescence change overlays the change in tail-current shape, a change that has been shown previously to correlate with the mode shift (Männikkö et al., 2005). Inset, Enlargement of the current during the step to +50 $\mathrm{mV}$, from $\boldsymbol{E}$. Note that in $\boldsymbol{C}, \boldsymbol{D}$, and $\boldsymbol{F}$, the fluorescence signal has been inverted for a direct kinetic comparison with the current traces.

channels are mainly in mode I and close with a fast, singleexponential time course, whereas after longer prepulses, HCN channels shift to mode II and close with a slower, sigmoidal time course (Männikkö et al., 2005; Elinder et al., 2006). As shown in Figure $3 F$, the time constant for the fluorescence change (101.6 \pm $2.2 \mathrm{~ms} ; n=5)$ correlated well with the time course of the changes in tail currents $(109.0 \pm 3.8 \mathrm{~ms} ; n=5)$ and was slower than channel activation ( $32.7 \pm 6.3 \mathrm{~ms} ; n=5 ; p<0.001)$, indicating that, in spHCN channels, the region of $\mathrm{S} 4$ around $326 \mathrm{C}$ undergoes conformational changes mainly during the mode shift. These data show that a fluorophore attached to 326C reports on conformational changes during the mode shift and that inactivation is slower than the mode shift. Therefore, we conclude that the mode shift is not caused by cAMP-dependent inactivation. However, our data leave open the possibility that the mode shift, in the absence of cAMP, leads to additional conformational changes that cause inactivation. Thus, the mode shift and inactivation are kinetically distinct processes, but the conformational changes that occur during the mode shift may be a prerequisite for inactivation.

\section{Consecutive S4 residues report on the mode shift}

The fluorescence change reported by the fluorophore attached to 326C could be attributable to a conformational change by S4 itself or by a conformational change of another channel domain that moves relative to $326 \mathrm{C}$, thereby quenching the fluorescence. To test whether $\mathrm{S} 4$ movement or a movement of another domain toward S4 is responsible for the fluorescence quenching, we introduced cysteines, one at a time, at consecutive positions in S4. If S4 is an $\alpha$ helix, then consecutive residues should point toward different directions and sample different environments around S4. If another domain moves toward S4 during the mode shift, then only fluorophores attached to residues that face that domain would be expected to display fluorescence changes. Conversely, if S4 moves during the mode shift, then fluorophores attached to residues facing all directions would be expected to display fluorescence changes.

In Figure 4, ionic currents and fluorescence signals are shown for three consecutive S4 residues: 324C-326C. The major fluorescent components for $324 \mathrm{C}$ and $325 \mathrm{C}$ channels were similar to the fluorescence from 326C channels in that they were slower than channel opening, suggesting that the fluorescence from the three most $\mathrm{N}$-terminal residues $(324 \mathrm{C}-326 \mathrm{C})$ track the mode shift (Fig. 4). To directly test whether the slow fluorescence signal from 324C during large hyperpolarizations correlated temporally with the mode shift, we measured the time course of the change in the tail currents after activating prepulses of increasing duration in Alexa-488-labeled 324C channels. The time constant for the fluorescence change for $324 \mathrm{C}(211.7 \pm 26.6 \mathrm{~ms} ; n=6)$ correlated well with the time course of the change in tail currents (216.7 \pm $14.9 \mathrm{~ms} ; n=6)$ and was slower than channel activation (28.1 \pm $3.9 \mathrm{~ms} ; n=5 ; p<0.0001)$. Similarly, the fluorescence time course for $325 \mathrm{C}(366.7 \pm 48.7 \mathrm{~ms} ; n=5)$ was slower than channel activation (75.6 $\pm 16.2 \mathrm{~ms} ; n=5 ; p<0.005)$, suggesting that the fluorescence reported on the mode shift. Unfortunately, the ionic currents from 325C channels activated slowly and at more negative potentials, necessitating very long hyperpolarizing voltage steps to activate these channels. The long hyperpolarizing voltage steps also activated endogenous currents that contaminated the tail currents, preventing a direct comparison between the fluorescence and the changes in the tail currents for 325C channels. However, the qualitative similarity of the kinetic relationship between fluorescence and the ionic current for 326C and 325C channels suggests that the slow fluorescence from 325C channels also reported on the mode shift (Fig. 4). The correlation between the fluorescence changes and the mode shift shows that the region of S4 containing residues 324C-326C undergoes a conformational change during the mode shift in spHCN channels. In contrast, fluorescence from a more internal S4 residue presumably reported on the fast-gating charge movements preceding channel opening (Fig. $2 G$ ), suggesting that the mode-shift movement of $\mathrm{S} 4$ is temporally distinct from the gating-charge movement of S4.

\section{The mode shift alters the kinetics of synaptic responses} Our fluorescence data show that conformational changes of outer S4 provide a structural correlate to the mode shift in HCN channel currents. This strongly supports our previous proposal that 

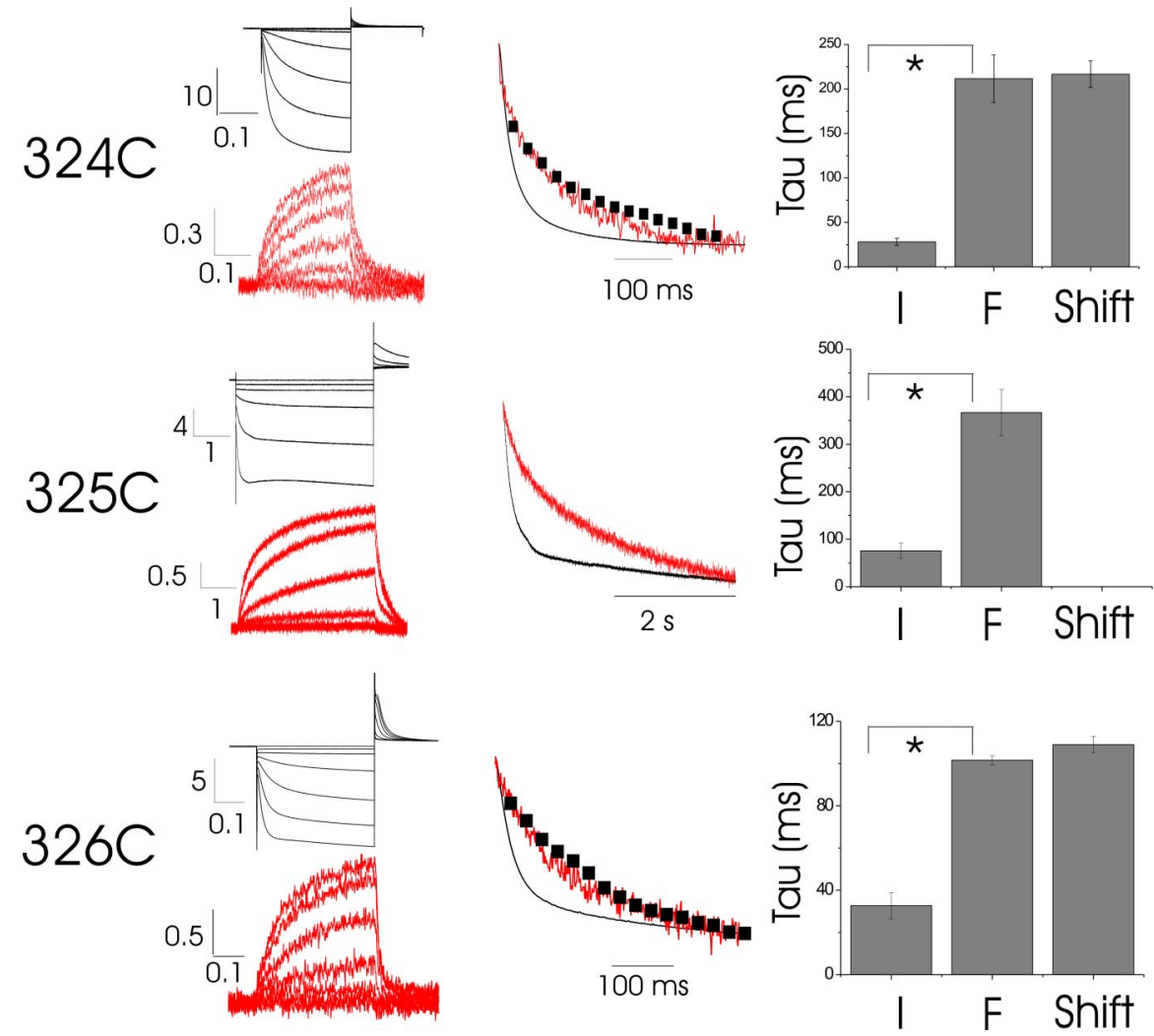

Figure 4. Fluorescence from consecutive $\mathrm{N}$-terminal $\mathrm{S4}$ residues report on the mode shift. Left, Representative current (top) and fluorescence (bottom) records for Alexa-488 C5-maleimide-labeled residues 324C-326C. Channels were held at $0 \mathrm{mV}$ and then stepped to negative potentials $(0$ to $-140 \mathrm{mV})$, followed by a step to $+50 \mathrm{mV} . x$ - and $y$-axis calibration bars indicate time (seconds) and current (microamperes), respectively. Note the different timescale for $325 \mathrm{C}$. Center, Normalized current (black) and fluorescence (red) traces during steps to $-120 \mathrm{mV}$. Black squares indicate the time course of the mode shift for each Alexa-488labeled mutant, measured as the time to half-decay of the tail currents as in Figure $3 F$. Right, Average time constants for channel activation (I), fluorescence (F), and the mode shift (Shift). ${ }^{*} p<0.01$, Student's $t$ test.
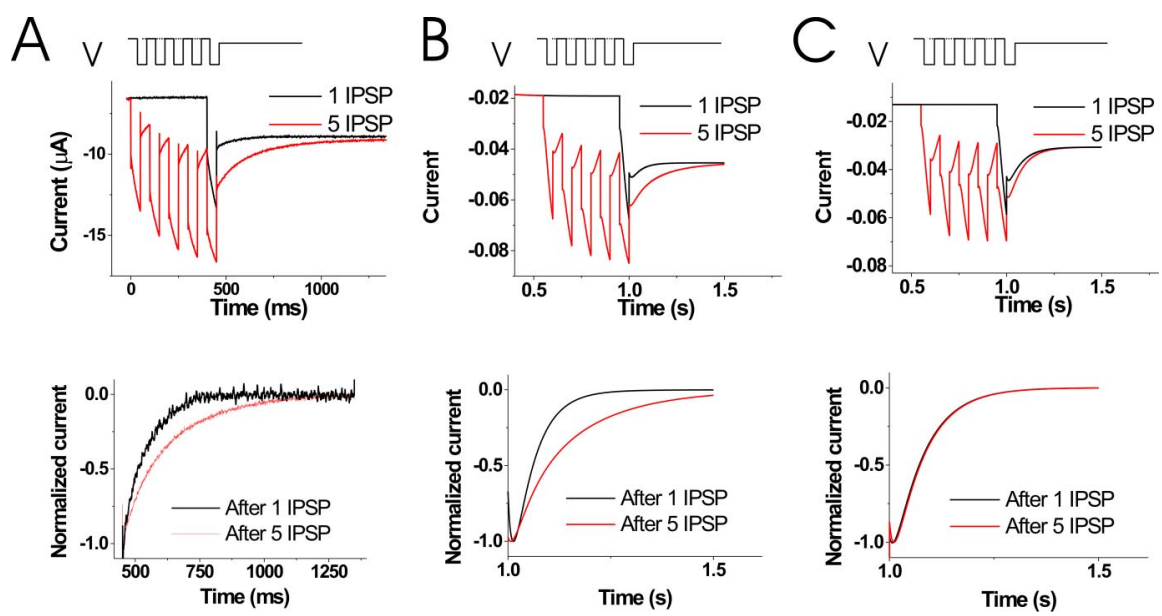

Figure 5. Mode shift in mammalian HCN channels affects response to trains of IPSPs. $A, A$ train of negative voltage pulses (10 $\mathrm{Hz}, \Delta V$ of $-20 \mathrm{mV}$ ), simulating a train of IPSPs, applied to an oocyte expressing HCN1 channels with deleted CNBD (see Materials and Methods). The oocyte was held at $-60 \mathrm{mV}$ before the train of IPSPs. After one IPSP, the tail currents were faster than after a train of five IPSPs. Tail potential of $-64 \mathrm{mV}$. Bottom, Normalized tail currents showing a kinetic change in the decay of the HCN1 currents after longer trains of IPSPS. B, C, Computer simulations of model HCN channels (similar to model 1; Materials and Methods). Top, Simulated currents in response to one simulated IPSP (black line) or a train of five simulated IPSPs (red line) $(10 \mathrm{~Hz}$, $\Delta V$ of $-20 \mathrm{mV}$ ) from model HCN channels with mode shift $(\boldsymbol{B})$ and without mode shift ( $\boldsymbol{C}$ (i.e., channels always in mode I). The $y$-axis is an arbitrary current scale. Inset, Voltage protocol used. Bottom, Normalized tail currents showing a kinetic change in the decay of the HCN currents after longer trains of IPSPs for the model with mode shift, suggesting that the change in the HCN1 tail current kinetics is attributable to the mode shift. both spHCN channels and the mammalian HCN channels undergo a mode shift and that dynamic voltage dependence is a fundamental property of HCN channels (Männikkö et al., 2005; Elinder et al., 2006). It is therefore important to test what effect the mode shift has on the functional roles that HCN channels play in physiological systems. We have shown previously by modeling that the mode shift plays an important role in stabilizing the rhythmic firing of pacemaker cells (Männikkö et al., 2005; Elinder et al., 2006). In addition to their role as pacemaker channels, it has been shown that HCN channels are expressed in dendrites and that modulation of HCN channels can alter the integration and the kinetics of synaptic responses (Magee, 1998; Magee and Carruth, 1999; Wang et al., 2002). We here wanted to test whether the mode shift also would affect synaptic responses.

We simulated IPSPs on oocytes expressing mammalian HCN1 channels, which have been shown to modulate synaptic integration in dendrites of hippocampal and Purkinje neurons (Magee, 1998; Magee and Carruth, 1999; Santoro and Tibbs, 1999; Nolan et al., 2003). The HCN channel current induced by different simulated trains of IPSPs was recorded (Fig. 5A). The kinetics of the HCN1 tail currents (i.e., the closing of the channels) were slower after a train of five IPSPs ( $\tau$ of $221 \pm 42 \mathrm{~ms} ; n=3$ ) than after a single IPSP ( $\tau$ of $115 \pm 20 \mathrm{~ms} ; n=3 ; p<0.05$ ). Changes in the kinetics of tail currents have been shown previously to be correlated with the mode shift in HCN channels, suggesting that the changes in the tail currents after different trains of IPSP are attributable to the mode shift (Männikkö et al., 2005). We tested this hypothesis in computer simulations using a HCN channel model with a mode shift (BrueningWright, Elinder, and Larsson, unpublished observations). The model HCN channel with a mode shift also displayed a slowing of the tail currents after longer trains of IPSP (Fig. 5B). However, when the mode shift was eliminated from the model HCN channels, then the kinetics of the tail currents were independent of the number of IPSPs (Fig. 5C). This is consistent with the idea that the kinetic changes in $\mathrm{HCN} 1$ tail currents after different trains of IPSPs are attributable to the mode shift. The mode shift thus serves to shorten the duration of the hyperpolarization caused by IPSPs or to induce a larger afterdepolarization (ADP) and, therefore, possibly alter the integration of trains of synaptic inputs (Fig. 6). In Figure 6, we simulated 
A

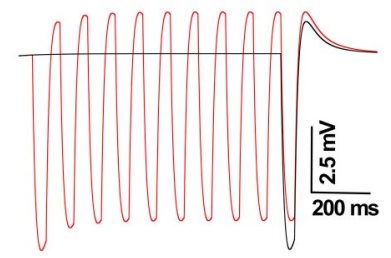

B

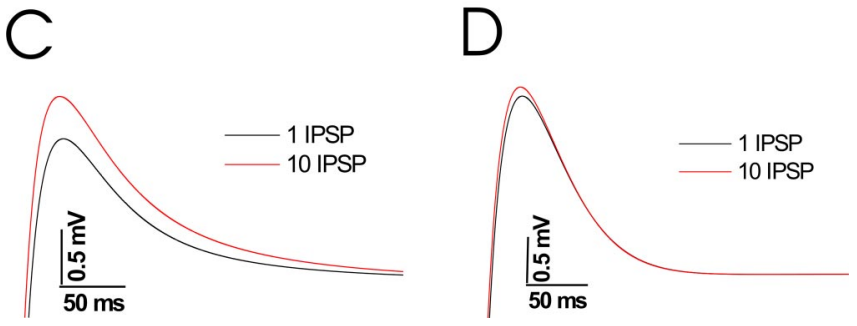

Figure 6. The mode shift induces an integration effect on the ADP after IPSPs. Simulations of the voltage response to 1 or 10 IPSPs ( $10 \mathrm{~Hz}, 20 \mathrm{pA})$ in a simple passive cell (i.e., below threshold; see Materials and Methods) with $\mathrm{HCN}$ channels with a mode shift $(\boldsymbol{A})$ or $\mathrm{HCN}$ channels without any mode shift $(\boldsymbol{B})$. The HCN channels create a sag in the IPSP response and an ADP after the IPSPs. Enlargement of the ADPs after the IPSP(s) in the model with the mode shift ( $\boldsymbol{C}$; from $\boldsymbol{A}$ ) and in the model without the mode shift $(\boldsymbol{D} ;$ from $\boldsymbol{B})$. In the model with a mode shift, the size of the ADP is dependent on the number of IPSP preceding the $\operatorname{ADP}(\boldsymbol{C})$. In the model without a mode shift, the ADP is not very dependent on the number of IPSPs (D).

IPSPs under nonvoltage-clamp conditions in a simple model cell with HCN channels (Methods and Materials). The simulations show the sag in the IPSP and the rebound potential (or ADP) after the IPSP, typical for cells expressing HCN channels. This is attributable to the slow opening and closing of HCN channels during and after the IPSP. For HCN channels with a mode shift, the size of the ADP was dependent on the number of IPSPs preceding the ADP. The ADP was smaller after only one IPSP and larger after several IPSPs (Fig. 6A,C). For HCN channels without a mode shift, the size of the ADP was basically independent of the number of IPSPs preceding the ADP (Fig. $6 B, D$ ). The actual effect that the mode shift has in a particular neuron would depend on the size, shape, and frequency of the synaptic inputs, the density of HCN channels and other ion channels present in the dendrite, and the electrotonic properties of the cell. This would have to be determined in future experiments in more physiological systems.

\section{Discussion}

Our results show that, during the mode shift in HCN channels, there is a conformational change in or around S4. Because fluorophores attached to all sides of the S4 helix (324C-326C) reported on this conformational change (Fig. 4), we propose that either it is $S 4$ that moves during the mode shift, not another domain (e.g., S5) that moves relative to S4, or the protein region surrounding S4 collapses, causing the fluorescence changes all around S4. The slow conformational change in S4 during the mode shift may be triggered by the relatively fast $S 4$ movement during gating charge translocation and/or the subsequent channel opening. Our data rule out models in which the mode shift is caused solely by a stabilizing conformational change in the pore domain that indirectly shifts the voltage dependence of the channel. This type of stabilizing mechanism does not predict a fluorescence change at the voltage sensor that would correlate with the mode shift; this mechanism only predicts a change in the kinetics and voltage dependence of the gating-charge movement of 44 (Fig. $1 D$ ). Instead, our results suggest that $\$ 4$ plays an active role in the conformational changes during the mode shift.

In addition to the movement of S4 during the mode shift reported here, conformational changes in the pore domain may also be important for the mode shift. In a previous study (Männikkö et al., 2005), we showed that both permeating $\mathrm{K}^{+}$and the pore blocker $\mathrm{Cs}^{+}$strongly affect the rate of the mode shift in mammalian HCN channels, suggesting that these ions modulate conformational changes in the pore by occupying the pore. For HCN1 channels, the mode shift was slowed more than threefold in high external $\mathrm{K}^{+}$concentrations compared with low external $\mathrm{K}^{+}$concentrations. Supplementing the high $\mathrm{K}^{+}$solution with $\mathrm{Cs}^{+}$restored the faster rate of mode shift, suggesting that $\mathrm{Cs}^{+}$ prevents $\mathrm{K}^{+}$from reaching its binding site in the pore (Männikkö et al., 2005). Unfortunately, $\mathrm{Cs}^{+}$and $\mathrm{K}^{+}$do not modulate the mode shift in spHCN channels (Männikkö et al., 2005; Elinder et al., 2006), so additional experiments on mammalian $\mathrm{HCN}$ channels are required to test whether $\mathrm{Cs}^{+}$also affects the conformational changes in $\mathrm{S} 4$ during the mode shift.

We found that $\mathrm{N}$-terminally located $\mathrm{S} 4$ residues (324C, 325C, and 326C) report on conformational changes during the mode shift but not on the gating-charge movements, consistent with previous accessibility studies (Fig. $2 A$ ) that showed a stateindependent accessibility for $\mathrm{N}$-terminal $\mathrm{S} 4$ residues (residues 326-332 in spHCN channels) (Männikkö et al., 2002; Bell et al., 2004; Vemana et al., 2004). In contrast, residues in the middle and C-terminal end of S4 (residues 333-349 in spHCN channels) are accessible in a state-dependent manner (Männikkö et al., 2002; Bell et al., 2004; Vemana et al., 2004), suggesting that these residues are the voltage-sensing charges that undergo the transmembrane movement that triggers channel opening. The mode shift itself exhibits little, if any, voltage dependence (Elinder et al., 2006), suggesting no large inward movement of the gating charges during the mode shift. In addition, there is no large slow component in the gating currents at hyperpolarized potentials (Männikkö et al., 2005), also suggesting that there is no large inward movement of the gating charges during the mode shift. We have not been able to directly label the charge-carrying part of S4 (residues 335-349) with fluorophores, and, therefore, we have not been able to directly measure the kinetics of movements of this part of S4.

We propose the following model for the conformational changes during the mode shift (Fig. 7). After the initial inward gating-charge movement of $S 4$ and the opening of the activation gate, slower conformational changes occur both in and around S4 and in the deeper regions of the pore. We propose that S4 undergoes a lateral or tilt movement during the mode shift, which would not significantly contribute to the gating-charge movement. These slower conformational changes stabilize S4 in its inward position and the activation gate in the open position, by strengthening bonds to nearby domains (e.g., adding hydrogen bonds or increasing charge-charge interactions between $\mathrm{S} 4$ and S2 charges). The extra energy in these bonds necessitates additional electrical energy to break these bonds to move $S 4$ back to its outward position and close the activation gate. This additional electrical energy causes a shift in the voltage dependence of the gating-charge movement to more depolarized potentials, resulting in the shift in the voltage dependence associated with the mode shift. The mechanism driving the mode shift and the type of conformational change that occurs in S4 remain to be determined.

Previous studies have shown that the CNBD affects gating in HCN channels (Wainger et al., 2001; Wang et al., 2001). How- 


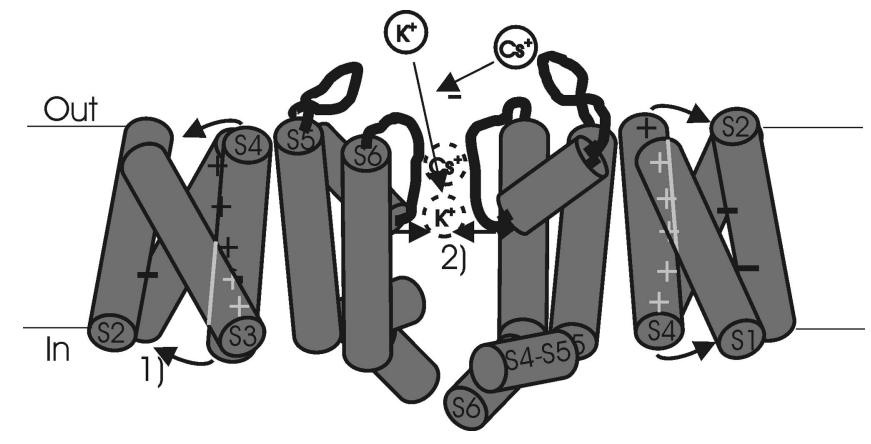

Figure 7. Model for pore and voltage-sensor movement during mode shift in HCN channels. The transmembrane domains of HCN were placed after homology modeling with the crystal structure of Kv1.2 channel (Long et al., 2005). Only two of the four pore domains and voltagesensing domains are shown. We propose the following: (1) S4 undergoes a conformational change during the mode shift that brings the $\$ 4$-positive charges closer to one or both of the two S2-negative charges, thereby stabilizing the inward position of S4. In addition, a second (2) conformational change occurs in the deeper regions of the pore that allosterically influence the conformational changes in S4. The conformational change of the pore is modulated by $\mathrm{K}^{+}$that binds deep in the selectivity filter and thereby prevents the conformational change. Externa $\mathrm{Cs}^{+}$can prevent $\mathrm{K}^{+}$binding by binding to a more external site in the pore.

ever, the mode shift is not directly affected by the CNBD. First, the mode shift occurs in channels in which we have removed the C terminus, including the entire $\mathrm{CNBD}$ and the $\mathrm{S} 6-\mathrm{CNBD}$ linker (Männikkö et al., 2005), and the mode shift occurs in channels with nonfunctional cyclic nucleotide-binding sites (Elinder et al., 2006). Second, the fluorescence change from $326 \mathrm{C}$ channels has a similar time course whether or not the CNBD has been mutated (Fig. 3). The R620G mutation in the CNBD causes a large shift in the sensitivity to cyclic nucleotides, as indicated by the inactivation of R620G channels. If the CNBD were affecting the mode shift, we would expect the R620G mutation to dramatically alter the kinetics of the $326 \mathrm{C}$ fluorescence. Therefore, we propose that the mode-shift conformational changes are limited to the voltage-sensing and pore domains.

The shift in the voltage dependence induced by the mode shift is reminiscent of the voltage shift in gating-charge movement that has been associated with slow inactivation in depolarizationactivated channels. For example, C-type inactivation in Shaker K channels has been correlated with a $Q(V)$ shift (Olcese et al., 1997), similar to the $Q(V)$ shifts in HCN channels during the mode shift (Männikkö et al., 2005). Moreover, for Shaker channels, slow inactivation is modulated by external $\mathrm{K}^{+}$in a manner similar to the way in which the mode shift is modulated in $\mathrm{HCN}$ channels. Therefore, we suggest that the conformational changes during the mode shift in HCN channels are similar to the conformational changes seen in Shaker channels during slow inactivation. In support of this hypothesis, previous VCF studies of Shaker channels have shown that N-terminal S4 residues report on conformational changes that correlate with C-type inactivation (Loots and Isacoff, 1998; Gandhi et al., 2000; Loots and Isacoff, 2000; Pathak et al., 2005), similar to what we have shown in this study for $\mathrm{N}$-terminal $\mathrm{S} 4$ residues in HCN channels. However, in contrast to Shaker channels, the $Q(V)$ shifts in $\mathrm{HCN}$ channels are not associated with the closing of an inactivation gate. Interestingly, the closing of the inactivation gate is not a necessity for the $Q(V)$ shifts in Shaker channels either, because a mutation in the pore domain enables Shaker channels to undergo the $Q(V)$ shift without closing the inactivation gate (Olcese et al., 2001). This suggests that the relatively slow conformational changes in S4 are not closely coupled to the conformational changes in the pore during inactivation; rather, slow S4 changes may be allosterically modulated by the conformational changes in the pore in Shaker K channels. Similarly, for HCN channels, we suggest that the slower conformational changes in S4 are not strongly coupled to conformational changes in the pore, although they may be modulated by the conformational changes in the pore and the ion occupancy of the pore.

We have here identified a slow conformational change that we believe is intrinsic to the S4 domain of HCN channels. The slow conformational change correlates with the mode shift in $\mathrm{HCN}$ channel voltage dependence, suggesting that $\mathrm{S} 4$ undergoes a conformational change during the mode-shift transition. This structural correlation to the mode shift strongly supports our previous proposal that HCN channels undergo a mode shift and that dynamic voltage dependence is a fundamental property of $\mathrm{HCN}$ channels (Männikkö et al., 2005). These findings are a first step toward understanding the molecular mechanism of the mode shift, which has been shown to be important in preventing the development of arrhythmia during pacemaking in models of the sino-atrial node in the heart (Männikkö et al., 2005). The mode shift imparts a short-term, activity-dependent memory to HCN channels, which we propose could modify integration of synaptic responses and contribute to the regulation of rhythmic firing patterns (Wang et al., 2002; Männikkö et al., 2005; Elinder et al., 2006). It has been shown previously that HCN channels modulate synaptic integration in neurons (Magee, 1998; Magee and Carruth, 1999; Nolan et al., 2003). However, the physiological role that the mode shift plays in synaptic integration remains to be tested in physiological systems. Additional experiments are needed to determine whether the mode shift is a property unique to $\mathrm{HCN}$ channels, perhaps adopted to suit their pacemaking function (Männikkö et al., 2005), or whether similar mode shifts in the voltage dependence occur in other members in the superfamily of voltage-gated ion channels.

\section{References}

Bell DC, Yao H, Saenger RC, Riley JH, Siegelbaum SA (2004) Changes in local S4 environment provide a voltage-sensing mechanism for mammalian hyperpolarization-activated HCN channels. J Gen Physiol 123:5-19.

Cha A, Bezanilla F (1997) Characterizing voltage-dependent conformational changes in the Shaker $\mathrm{K}^{+}$channel with fluorescence. Neuron 19:1127-1140.

Chen S, Wang J, Siegelbaum SA (2001) Properties of hyperpolarizationactivated pacemaker current defined by coassembly of HCN1 and HCN2 subunits and basal modulation by cyclic nucleotide. J Gen Physiol 117:491-504.

DiFrancesco D (1993) Pacemaker mechanisms in cardiac tissue. Annu Rev Physiol 55:455-472.

Elinder F, Männikkö R, Pandey S, Larsson HP (2006) Mode shifts in the voltage gating of the mouse and human HCN2 and HCN4 channels. J Physiol (Lond) 575:417-431.

Gandhi CS, Loots E, Isacoff EY (2000) Reconstructing voltage sensor-pore interaction from a fluorescence scan of a voltage-gated $\mathrm{K}^{+}$channel. Neuron 27:585-595.

Gauss R, Seifert R, Kaupp UB (1998) Molecular identification of a hyperpolarization-activated channel in sea urchin sperm. Nature 393:583-587.

Hille B, Armstrong CM, MacKinnon R (1999) Ion channels: from idea to reality. Nat Med 5:1105-1109.

Johnson Jr JP, Zagotta WN (2005) The carboxyl-terminal region of cyclic nucleotide-modulated channels is a gating ring, not a permeation path. Proc Natl Acad Sci USA 102:2742-2747.

Larsson HP, Tzingounis AV, Koch HP, Kavanaugh MP (2004) Fluorometric measurements of conformational changes in glutamate transporters. Proc Natl Acad Sci USA 101:3951-3956.

Li M, Lester HA (2002) Early fluorescence signals detect transitions at mammalian serotonin transporters. Biophys J 83:206-218. 
Long SB, Campbell EB, Mackinnon R (2005) Crystal structure of a mammalian voltage-dependent Shaker family $\mathrm{K}^{+}$channel. Science 309:897-903.

Loots E, Isacoff EY (1998) Protein rearrangements underlying slow inactivation of the Shaker K ${ }^{+}$channel. J Gen Physiol 112:377-389.

Loots E, Isacoff EY (2000) Molecular coupling of S4 to a $\mathrm{K}^{+}$channel's slow inactivation gate. J Gen Physiol 116:623-636.

Ludwig A, Budde T, Stieber J, Moosmang S, Wahl C, Holthoff K, Langebartels A, Wotjak C, Munsch T, Zong X, Feil S, Feil R, Lancel M, Chien KR, Konnerth A, Pape HC, Biel M, Hofmann F (2003) Absence epilepsy and sinus dysrhythmia in mice lacking the pacemaker channel HCN2. EMBO J 22:216-224.

Magee JC (1998) Dendritic hyperpolarization-activated currents modify the integrative properties of hippocampal CAl pyramidal neurons. J Neurosci 18:7613-7624.

Magee JC, Carruth M (1999) Dendritic voltage-gated ion channels regulate the action potential firing mode of hippocampal CA1 pyramidal neurons. J Neurophysiol 82:1895-1901.

Männikkö R, Elinder F, Larsson HP (2002) Voltage-sensing mechanism is conserved among ion channels gated by opposite voltages. Nature 419:837-841.

Männikkö R, Pandey S, Larsson HP, Elinder F (2005) Hysteresis in the voltage dependence of HCN channels: conversion between two modes affects pacemaker properties. J Gen Physiol 125:305-326.

Mannuzzu LM, Moronne MM, Isacoff EY (1996) Direct physical measure of conformational rearrangement underlying potassium channel gating. Science 271:213-216.

Nolan MF, Malleret G, Lee KH, Gibbs E, Dudman JT, Santoro B, Yin D, Thompson RF, Siegelbaum SA, Kandel ER, Morozov A (2003) The hyperpolarization-activated $\mathrm{HCN} 1$ channel is important for motor learning and neuronal integration by cerebellar Purkinje cells. Cell 115:551-564.

Olcese R, Latorre R, Toro L, Bezanilla F, Stefani E (1997) Correlation between charge movement and ionic current during slow inactivation in Shaker $\mathrm{K}^{+}$channels. J Gen Physiol 110:579-589.

Olcese R, Sigg D, Latorre R, Bezanilla F, Stefani E (2001) A conducting state with properties of a slow inactivated state in a shaker $\mathrm{K}^{+}$channel mutant. J Gen Physiol 117:149-164.
Pape HC (1996) Queer current and pacemaker: the hyperpolarizationactivated cation current in neurons. Annu Rev Physiol 58:299-327.

Pathak M, Kurtz L, Tombola F, Isacoff E (2005) The cooperative voltage sensor motion that gates a potassium channel. J Gen Physiol 125:57-69.

Robinson RB, Siegelbaum SA (2003) Hyperpolarization-activated cation currents: from molecules to physiological function. Annu Rev Physiol 65:453-480.

Rothberg BS, Shin KS, Phale PS, Yellen G (2002) Voltage-controlled gating at the intracellular entrance to a hyperpolarization-activated cation channel. J Gen Physiol 119:83-91.

Santoro B, Tibbs GR (1999) The HCN gene family: molecular basis of the hyperpolarization-activated pacemaker channels. Ann NY Acad Sci 868:741-764.

Schulze-Bahr E, Neu A, Friederich P, Kaupp UB, Breithardt G, Pongs O, Isbrandt D (2003) Pacemaker channel dysfunction in a patient with sinus node disease. J Clin Invest 111:1537-1545.

Shin KS, Maertens C, Proenza C, Rothberg BS, Yellen G (2004) Inactivation in $\mathrm{HCN}$ channels results from reclosure of the activation gate: desensitization to voltage. Neuron 41:737-744.

Ueda K, Nakamura K, Hayashi T, Inagaki N, Takahashi M, Arimura T, Morita H, Higashiuesato Y, Hirano Y, Yasunami M, Takishita S, Yamashina A, Ohe T, Sunamori M, Hiraoka M, Kimura A (2004) Functional characterization of a trafficking-defective HCN4 mutation, D553N, associated with cardiac arrhythmia. J Biol Chem 279:27194-27198.

Ulens C, Siegelbaum SA (2003) Regulation of hyperpolarization-activated HCN channels by cAMP through a gating switch in binding domain symmetry. Neuron 40:959-970.

Vemana S, Pandey S, Larsson HP (2004) S4 movement in a mammalian HCN channel. J Gen Physiol 123:21-32.

Wainger BJ, DeGennaro M, Santoro B, Siegelbaum SA, Tibbs GR (2001) Molecular mechanism of cAMP modulation of HCN pacemaker channels. Nature 411:805-810.

Wang J, Chen S, Siegelbaum SA (2001) Regulation of hyperpolarizationactivated $\mathrm{HCN}$ channel gating and cAMP modulation due to interactions of $\mathrm{COOH}$ terminus and core transmembrane regions. J Gen Physiol 118:237-250.

Wang J, Chen S, Nolan MF, Siegelbaum SA (2002) Activity-dependent regulation of HCN pacemaker channels by cyclic AMP: signaling through dynamic allosteric coupling. Neuron 36:451-461. 